\title{
Bemerkungen zur Feinstgliederung und zum Kalkgehalt des Lösses
}

\author{
Von Karl Brunnacker, München
}

Mit 1 Abbildung und 2 Tabellen im Text

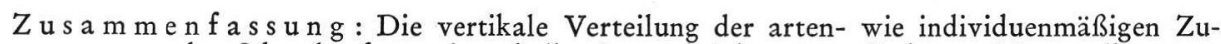
sammensetzung der Schneckenfauna innerhalb eines scheinbar ungegliederten Lösses gibt unter Mitberücksichtigung der Rhizosolenien (Wurzelröhrchen), des Kalkgehaltes und der Korngrößenverteilung Hinweise auf wechselnde Sedimentationsbedingungen. Ein wichtiger Anzeiger für die Bodendynamik während der Lößablagerung ist der Kalkgehalt des Lösses, der durch mehrere Faktoren bestimmt wird (Zusammensetzung des Ausblasungsgesteins, regionalklimatische und standörtliche Gegebenheiten, die Sedimentationsgeschwindigkeit und Körnung des Lösses).

$\mathrm{Summary}$ : The vertical distribution of the species as well as the composition of individuals of the mollusc fauna within an apparently non-divided loess, gives some references to varying conditions of sedimentation, with the aid of Rhizosoleniens, calcium carbonate content and distribution of grain sizes. An important indicator for the dynamics of soils during the deposition of loess, is the calcium carbonate content of the loess, determined by several factors (composition of wind-eroded rocks, regional-climatic and local circumstances, speed of sedimentation and granulation of the loess).

$\mathrm{R}$ é $\mathrm{s}$ u mé : La distribution verticale des espèces aussi bien que la composition des individus de la faune de molluscs à l'intérieur d'un loess en apparence non-dividé, attire l'attention sur des conditions variées de sédimentation, à l'aide des Rhizosoléniens, de la teneur en carbonate de calcium et de la distribution du diamètre de grains. Un indicateur important pour la dynamique des sols pendant la déposition du loess, est la teneur en carbonate de calcium du loess, déterminée par plusieurs facteurs (composition des roches érodées du vent, conditions régionalesclimatiques et locales, vitesse de sédimentation et granulation du loess).

Die Großgliederung von Periglazialprofilen - nämlich die Unterscheidung interglazialer Böden (auf Löß: Parabraunerden, teils geköpft, teils in Pseudogley umgewandelt) und eiszeitlicher Ablagerungen (Fließerde und Löß) - macht heute unter Berücksichtigung einiger bodenkundlicher Gesichtspunkte in Süddeutschland keine größeren Schwierigkeiten mehr. Die Feingliederung der eiszeitlichen Ablagerungen und Böden innerhalb solcher Profile - Fließerde, Löß, interstadiale Böden (Tundren-Naßboden, brauner Verwitterungshorizont) - ist ebenfalls bei einiger Erfahrung an den Aufschlüssen noch möglich. Die bisher angewendeten Laboratoriumsmethoden bringen dazu lediglich Bestätigungen oder gewisse Ergänzungen (vgl. E. SснӧNHALS 1952, 1953, 1955, E. W. Guenther 1953a u. b, 1954, K. Brunnacker 1956b). Daneben hat es aber sicherlich noch kleinere Klima- und Sedimentationsschwankungen zumindest lokaler Art sowie standörtliche Eigenheiten gegeben, die im Profilbild nicht mehr zum Ausdruck kommen und die auch bei den bisherigen Untersuchungsmethoden kaum zu erkennen sind. Andererseits haben Untersuchungen über die Molluskenfauna in Lößprofilen (M. Brunnacker \& K. Brunnacker 1956) gezeigt, daß die Schneckenfauna unter Berücksichtigung des Standortes noch wenig genutzte Möglichkeiten zur Klärung von Feinheiten innerhalb der Profile bietet, ebenso wie die Rhizosolenien (Wurzelröhrchen); denn beispielsweise bilden sich bei feuchterem Klima infolge der weniger starken oder weniger häufigen Austrocknung des Bodens nicht so viele Wurzelröhrchen durch Kalkverkittungen als unter trockeneren Bedingungen, während andererseits die Schneckenfauna bei etwas feuchteren Verhältnissen besser vorankommt als bei trockeneren. Bei schnellerer Lößsedimentation werden sich aber ebenfalls weniger Rhizosolenien bilden als bei langsamer, auch die Fauna muß darauf irgendwie ansprechen. Unter Berücksichtigung solcher Gesichtspunkte und deren gemeinsamer Betrachtung muß es möglich sein, eine Feinstgliederung des Lösses zu erreichen. 
Die folgenden Ausführungen beschränken sich, soweit nicht anders angegeben, auf den Oberen Löß, der als jüngster Löß in Süddeutschland oben zum nacheiszeitlichen Boden verwittert ist und der im Liegenden durch den WI/II-Boden (meiner Arbeitsgliederung) begrenzt wird.

\section{Das Profil Estenfeld}

Das Profil Estenfeld nördlich Würzburg (vgl. K. Brunnacker 1957a) liegt an einem leicht nach Osten geneigten Oberhang. Über $80 \mathrm{~cm}$ Löß folgt der heute $0,8 \mathrm{~m}$ mächtige Boden. Damit vertritt der noch kalkreiche Löß etwa die Hälfte der ursprünglichen Lößmächtigkeit. Diesem Löß und den darunter liegenden Bildungen wurden von $15 \mathrm{zu} 15$ bzw. $20 \mathrm{~cm}$ als vorbereitende Untersuchung Proben entnommen und im bodenkundlichen Laboratorium des Bayer. Geologischen Landesamtes, München, auf Kalkgehalt und Korngrößenverteilung untersucht; von jeweils $500 \mathrm{~g}$ wurde innerhalb des Rückstandes über $0,2 \mathrm{~mm} \phi$ die Schneckenfauna durch meine Frau sowie die Zusammensetzung der Rhizosolenien bestimmt (Tab. I).

\begin{tabular}{|c|c|c|c|c|c|c|c|}
\hline \multirow[b]{2}{*}{$\begin{array}{l}\text { Tiefe } \\
\text { in } \mathrm{cm}\end{array}$} & \multirow[b]{2}{*}{$\begin{array}{c}\mathrm{CaCO}_{3} \\
\%\end{array}$} & \multicolumn{2}{|c|}{$\mathrm{mm} \phi$} & \multicolumn{3}{|c|}{ Zahl d. Schnecken pro $500 \mathrm{~g}$ Löß } & \\
\hline & & $\begin{array}{l}\langle 0,02 \\
\%\end{array}$ & $\begin{array}{c}\text { Rückstand } \\
>0,2 \%\end{array}$ & $\begin{array}{l}\text { Succinea } \\
\text { oblonga }\end{array}$ & $\begin{array}{l}\text { Pupilla } \\
\text { muscorum }\end{array}$ & $\begin{array}{l}\text { Vallonia } \\
\text { costata }\end{array}$ & \\
\hline $80-100$ & 16,1 & 21,3 & 1,2 & 1 & - & - & \multirow{5}{*}{ Oberer Löß (WII } \\
\hline $100-115$ & 18,5 & 19,4 & 2,0 & 7 & 1 & - & \\
\hline $115-130$ & 22,6 & 21,3 & 3,6 & 19 & 2 & 1 & \\
\hline $130-145$ & 21,9 & 20,6 & 4,0 & 13 & 1 & - & \\
\hline $145-160$ & 20,0 & 21,0 & 3,2 & 11 & 3 & - & \\
\hline $160-180$ & 1,0 & 25,0 & 1,2 & 4 & 1 & 1 & \multirow{2}{*}{$\begin{array}{l}\text { W I/II Brauner } \\
\text { Verwitterungs- } \\
\text { horizont }\end{array}$} \\
\hline $180-200$ & 2,0 & 24,9 & 2,4 & 2 & 1 & - & \\
\hline $200-220$ & 16,9 & 22,7 & 2,4 & 2 & 3 & - & \multirow{4}{*}{$\begin{array}{l}\text { Unterer Löß (WI } \\
\text { (mit Fließlöß- } \\
\text { einlagerungen) }\end{array}$} \\
\hline $220-240$ & 20,5 & 20,5 & 2,0 & 2 & 3 & - & \\
\hline $240-260$ & 16,3 & 24,4 & 2,4 & 2 & 6 & 2 & \\
\hline $260-280$ & 21,4 & 23,4 & 7,2 & 3 & 13 & 2 & \\
\hline
\end{tabular}

Tabelle I: Profil Estenfeld

Im Oberen Löß steigt der Kalkgehalt von 160 bis $115 \mathrm{~cm}$ Tiefe an. Unter Berücksichtigung auch der hier nicht im einzelnen angeführten Korngrößenverteilung kommt es gleichzeitig zu einer Kornverfeinerung. Die Probe $80-100 \mathrm{~cm}$ fällt infolge stärkerer Entkalkung deutlich heraus; die Probe 100-115 cm liegt noch innerhalb des den frischen Löß kennzeichnenden gesetzmäßigen Verhaltens, dürfte jedoch bereits durch schwache, die Körnung aber noch nicht beeinflussende Entkalkung gezeichnet sein (Abnahme der Rhizosolenien).

Die Fauna entspricht in ihrem armen Arten- und Individuenbestand den niederbayerischen Verhältnissen an vergleichbaren Standorten. Die Zusammensetzung der Rhizosolenien (vgl. Abb. 1 in M. Brunnacker \& K. Brunnacker 1956), innerhalb derer hier kleine stengelige Bildungen überwiegen, ließ mit der bisher angewendeten Methode der Abschätzung keine wesentlichen Unterschiede mehr erkennen, weshalb auf deren eingehendere Beschreibung verzichtet wird. Es kam bei diesem Profil weiter darauf an, die Beziehungen zwischen Kalkgehalt und Korngrößenverteilung zu überprüfen (vgl. E. Schönhals 1952) und die Tiefenwirkung der nacheiszeitlichen Bodenbildung unter den eigentlichen Verlehmungsbereich hinab zu fassen. Entsprechend E. SchöNHaLS (1952) fällt größerer Anteil an feineren Korngrößen mit hohem Kalkgehalt zusammen. Die Probe $80-100 \mathrm{~cm}$ gehört demnach noch in den Einflußbereich der Verwitterung. Die Probe $100-115 \mathrm{~cm}$ paßt sich den erwähnten Beziehungen bereits an, scheint aber ebenfalls noch etwas gestört zu sein (Abnahme der Rhizosolenien, s. unten). 


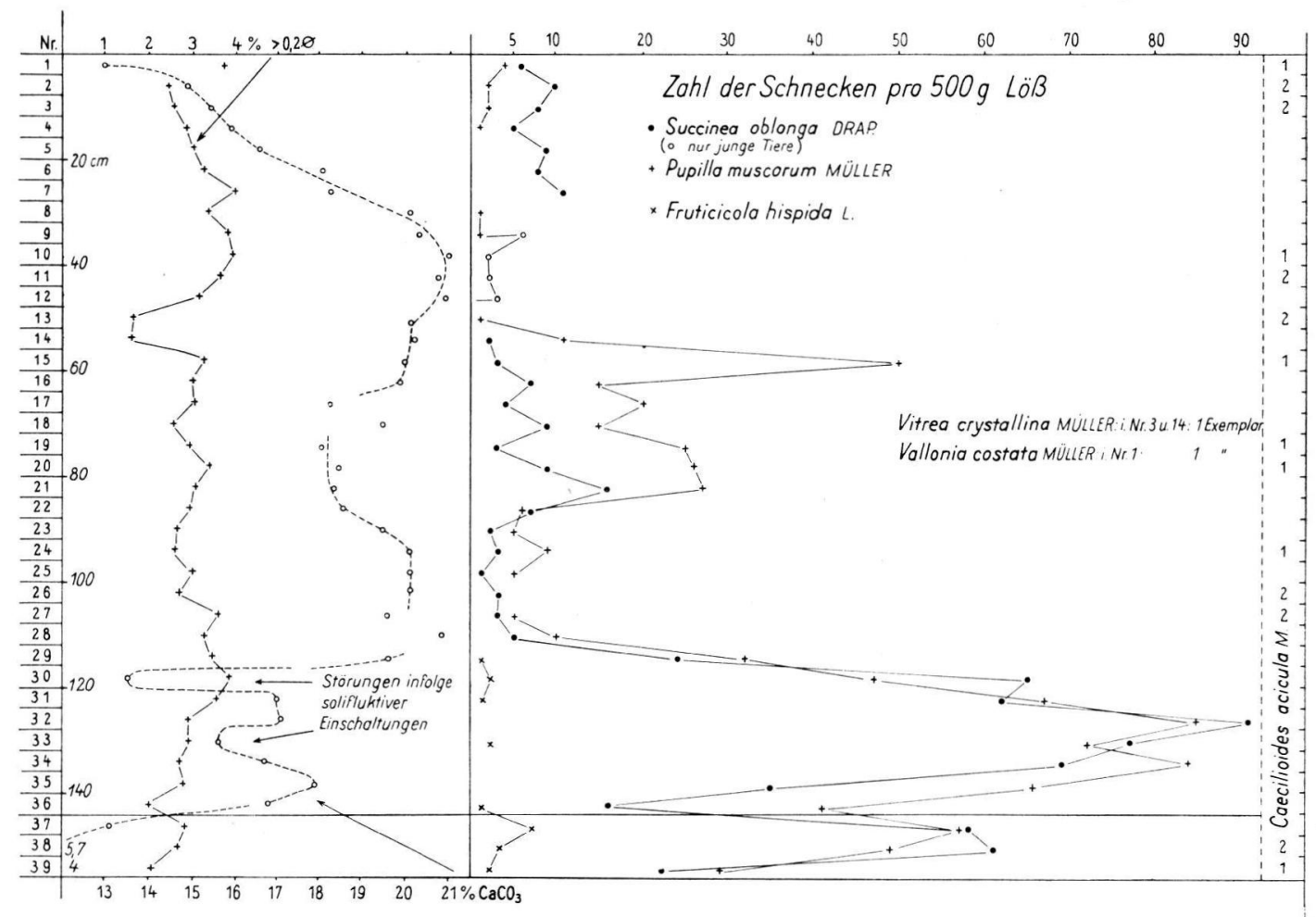

Abb. 1. Profil Kitzingen. Beziehung zwischen Rhizosolenien (=Rückstand $>0,2 \mathrm{~mm} \phi$ ), Kalkgehalt und Schneckenfauna im Oberen Löß.

\section{Das Profil Kitzingen}

Das Profil der Ziegelei Korbacher in Kitzingen (vgl. K. Brunnacker 1956a) liegt am Unterhang in ebenfalls etwa östlicher Exposition. Hier dürfen entsprechend den in Pfelling gemachten Feststellungen (M. Brunnacker \& K. Brunnacker 1956) günstigere Lebensbedingungen für die Schneckenfauna bei etwas mächtigerem, nicht verwittertem Löß erwartet werden. Es wurden die Proben von $4 \mathrm{zu} 4 \mathrm{~cm}$ entnommen (Abb. 1). Der wegerodierte nacheiszeitliche Waldboden (Parabraunerde) ist auch hier entsprechend ungestörten Bodenprofilen mit $\mathrm{rd} .1,0 \mathrm{~m}$ zu veranschlagen. Die Untergrenze des Lösses liegt im Bereich der Probe Nr. 37. Auch hier wurde, wie in Estenfeld, die Schneckenfauna bestimmt und zahlenmäßig erfaßt; von sämtlichen Proben wurde der Kalkgehalt und von einigen die Korngröße bestimmt.

Schon die allgemeine Abnahme der Zahl der Schnecken von unten nach oben deutet auf zunehmend ungüntigere Lebensbedingungen und zwar entsprechend niederbayerischen Profilen auf eine Entwicklungstendenz von kühl-,,feucht“ zu kalt-,trocken“ (Abb. 1). Dort hat sich - zumal bei größeren Individuenzahlen - gezeigt, daß das gemeinsame Auftreten von Succinea, Pupilla ind Fruticicola günstigere, insbesondere etwas feuchtere Zeitabschnitte bzw. Standorte anzeigt. Succinea, im wesentlichen allein und mit geringen Individuenzahlen, deutet dagegen auf kalte und trockenere Verhältnisse. ${ }^{1}$ )

1) Bei einem Vergleich der absoluten Zahlenwerte ist zu berücksichtigen, daß in Niederbayern jeweils rd. 1000, hier aber nur $500 \mathrm{~g}$ Löß geschlämmt wurden. - Caecilioides acicula MülLER, die sich tief im Boden vergräbt, dürfte nachträglich eingewandert sein (Abb. 1). 
In den Proben Nr. 37-39 sind Faunenreste aus dem WI/II, das auch in Kitzingen in der Fazies des braunen Verwitterungshorizontes ausgebildet ist, enthalten. Bei rezenten kalkarmen Böden sind die Schalen abgestorbener Schnecken korrodiert - teils als Folge der Verwitterung, teils infolge Anfressens durch lebende Schnecken zur Kalkaufnahme. Auch diese Schalen in Kitzingen sind angelöst; der Boden war also ursprünglich praktisch kalkfrei. Die erhaltenen Schalen stammen demgemäß von etwas eingegrabenen Tieren aus dem letzten Abschnitt des WI/II unmittelbar vor der erneut beginnenden Lößsedimentation; denn bei der Kalkauswaschung während des W I/II („brauner Verwitterungshorizont") sind ältere Schalen vollständig aufgelöst worden. Eine entsprechende Zusammensetzung der Fauna tritt zu Beginn der Lößablagerung auf (Nr. 36-30). - Zwischen Nr. 29 und 22 nimmt die Individuenzahl erheblich ab, wobei Pupilla etwas häufiger vorzukommen scheint als Succinea. - Zwischen Nr. 21 und 15 nimmit die Individuenzahl wieder zu mit einer deutlichen Dominanz der Pupilla; daraus darf wieder auf etwas günstigere Lebensbedingungen (etwas wärmeres Klima?) geschlossen werden. - Zwischen Nr. 14 und 8 treten die Schnecken dagegen stark zurück; Succinea herrscht zahlenmäßig vor - jedoch nur jugendlich abgestorbene Exemplare. Insgesamt waren die Lebensbedingungen jetzt sehr ungünstig. Zwischen Nr. 8 und 1 kommen die Schnecken wieder etwas häufiger, aber ebenfalls als individuenarme Succineenfauna vor. Die obersten Proben sind evtl. durch die nacheiszeitliche Verwitterung leicht gestört.

Die drei aus dem Oberen Löß von Pfelling untersuchten Proben (M. Brunnacker \& K. Brunnacker 1956) zeigen ebenfalls eine Konzentrierung der Fruticicola, aber auch der Succinea, auf den untersten Abschnitt. Pupilla entspricht zahlenmäßig der Succinea bzw. übertrifft sie weitaus. Anscheinend zeigen die Proben von Pfelling Ausschnitte einer ähnlichen vertikalen Differenzierung, wie sie für Kitzingen gefunden wurde. Es müßte deshalb möglich sein, mit Hilfe von Standardprofilen der Molluskenfauna, zumindest innerhalb enger begrenzter Gebiete und unter Berücksichtigung der standörtlichen Lage, Diskordanzen und Schichtlücken bzw. deren Fehlen, im Löß nachzuweisen. Das wäre insofern von Bedeutung, als manche - bodenkundliche Kriterien m. E. zu wenig berücksichtigende - Lößgliederungen derartigen Lücken innerhalb der Profile eine entscheidende Rolle zuspricht.

Die Rhizosolenien werden ihrer Menge nach durch den Rückstand über $0,2 \mathrm{~mm} \phi$ einigermaßen charakterisiert. Die Probe Nr. 1 ist durch kleine Eisenkonkretionen der nacheiszeitlichen Verwitterung beeinflußt, und bis etwa Nr. 6 liegt eine Störung durch teilweise Wegführung des die Röhrchen verkittenden Kalkes vor. Ein Rückgang der Rhizosolenien (bzw. des Rückstandes $>0,2 \mathrm{~mm} \phi$ ) ist z. T. mit einem Rückgang der Schneckenzahlen verbunden (Abb.1). Wo beide Werte sich rückläufig verhalten, deuten sie eine verhältnismäßig schnelle Lößsedimentation an; denn dadurch wurde die an sich spärliche Vegetation begraben bzw. deren sehr flacher Wurzelraum schnell höher gelegt und die Schneckenfauna vielieicht z. T. direkt durch die plötzliche Sedimentationsverstärkung, teils auf dem Weg über Vegetationsmangel gestört; auf jeden Fall wurde aber das zeitliche Verhältnis von Aufwuchs des Lösses und Faunendichte dabei verschoben.

Zeiten verlangsamter Sedimentation, insbesondere von $\mathrm{Nr} .36$ bis 30, aber auch von Nr. 21 bis 15 sind etwas weniger kalkreich²) als die Abschnitte mit schnellerer Sedimentation (29-22 und 14 und darïber). Schließlich folgen in den obersten Proben "sekundäre" Veränderungen im Zuge der Verwitterung nach Ablagerung des Lösses. Allerdings sind die Veränderungen der Kalkgehalte etwas tiefer verlagert als diejenigen, welche durch die Fauna und die Rhizosolenien angezeigt werden. Im Zuge solcher Kalkverlagerungen wurde auch der WI/II-Boden von oben nach unten abnehmend aufgekalkr.

2) Bei den Kalkgehalten muß mit geringen Analysenfehlern (Methode ScheibLER) gerechnet werden. $\mathrm{Ob}$ den Kleinschwankungen im Kalkgehalt (Nr. 28/27 und 18/17) eine besondere Bedeutung zukommt, bleibt deshalb vorläufig unbekannt. 
Störungen der Lößsedimentation sind außerdem durch schwache solifluktive Einschaltungen im untersten Teil des Lösses bedingt (z. B. in Nr. 33 und 30 durch Material des am Hang anfangs noch nicht von Löß eingedeckten braunen Verwitterungshorizontes). Doch darf eine derartige Mikrosolifluktion - noch dazu bei Beginn der jeweiligen Lößsedimentation - nicht überbewertet werden; die Solifluktion ist danach zu beurteilen, ob es sich in Beziehung zu einer bestimmten Zeiteinheit um geringfügige Verlagerungen, wie hier, oder um sehr kräftige und dementsprechend weiträumige Umlagerungen („Basisfließerde“ - K. BRunNACKer 1957a) handelt.

Eine Kombination dieser Angaben zeigt, daß die Lößanwehung schlagartig eingesetzt hat; dennoch war jhre Sedimentationsgeschwindigkeit anfänglich verhältnismäßig gering (Nr. 36-30), dann folgte eine sehr schnelle Anwehung mit gleichzeitigem Faunenwechsel (Nr. 29-22), darauf wieder Verlangsamung (Nr. 21-15), dann schnelle Sedimentation bzw. trocken-kaltes Klima mit Faunenwechsel (Nr. 14-8). Darüber ist die Deutung durch die Verwitterung erschwert. Der Faunenwechsel bei Nr. 29 (oder bei 21) und bei Nr. 12 weist auf Veränderungen des Klimas; hier müßten entsprechende Untersuchungen an noch besser geeigneten Profilen unter Berücksichtigung regionaler Gesichtspunkte angesetzt werden. Die Lößanwehung dagegen wurde in ihrer Geschwindigkeit durch lokale Gegebenheiten beeinflußt, wenngleich der Faunenwandel an Zeiten der Sedimentationsbeschleunigung gebunden zu sein scheint.

\section{Vergleich $z$ wischen Kitzingen und Estenfeld}

Die Proben in Estenfeld sind in einem zu weiten Abstand genommen worden. Insgesamt zeichnet sich hier dennoch wie in Kitzingen eine ähnliche Entwicklungstendenz zur Verarmung der Schneckenfauna nach oben zu ab, wobei jedoch als Folge der Geländelage ein etwas trockenerer Standort als in Kitzingen vorliegt. In Estenfeld setzt die Lößsedimentation etwas später als in Kitzingen ein (etwa ab Nr. 28 oder 21 in Kitzingen).

Dem entsprechen auch die sonstigen Geländebeobachtungen in Mainfranken: die Lößablagerung beginnt an den nach Osten exponierten Unterhängen, greift hangaufwärts, dann geht sie auf die ebenen Hochflächen über und erfaßt schließlich auch noch als dünne, heute durch die Bodenbildung völlig überprägte Decke die steilen nach Westen geneigten Lagen. Soweit der Löß nicht durch die nachfolgende Bodenbildung verändert ist, liegt sein Kalkgehalt bei geringerer Mächtigkeit im Bereich der Hochflächen etwas über dem der nach Osten exponierten. Hänge. Auch das spricht für eine Transgression der Lößfazies, die von den nach Ost exponierten Lagen ihren Ausgang nahm und die überall dort, wo sie einmal eingesetzt hat, etwa gleiche Aufwuchsmächtigkeiten zeigte.

\section{Bemerkungen zum Kalkgehalt des Lösses}

Im Profil Kitzingen wurde offen gelassen, inwieweit die Verteilung des Kalkgehaltes durch Wegführung und durch Verlagerung innerhalb des Profils bestimmt wird. Um diese Frage zu klären, muß etwas weiter ausgeholt werden. Bei Betrachtungen über den Kalkgehalt des Lösses sind regionale Unterschiede zu berücksichtigen, ferner an die Sedimentation gebundene und schließlich durch nachträgliche Verwitterung bedingte.

In Mainfranken liegt der Kalkgehalt des (Oberen) Lösses bei $20 \%$, in Südbayern bei 30-35\% und in den lokalen Lößlagern Mittelfrankens (nach H. LöвER 1932) bei nur $\mathrm{rd}$. 7\% . In Mittelfranken wird der Kalkgehalt vor allem materialbedingt sehr niedrig liegen, da hier die weiten Flächen des kalkarmen Sandsteinkeupers als Staublieferanten mitgewirkt haben. Auch ist der Löß hier etwas grobkörniger, so daß eine leichtere Auswaschung in Betracht zu ziehen ist. Zwischen Mainfranken und Südbayern kommen Materialunterschiede in Frage, daneben regionalklimatische, die sich selbst innerhalb Südbayerns andeuten; denn im Donaugebiet scheint der Kalkgehalt des Lösses 
etwas niedriger zu liegen als weiter im Süden, wo man an sich ein feuchteres Klima auch für die Lößzeiten erwarten darf. Bei heute rd. $900 \mathrm{~mm}$ Niederschlag verschwindet dann der Kalkgehalt im Löß sehr schnell (Decklehm). Im südlichen Südbayern ist der Niederterrassenschotter als Ausblasungsbereich viel kalkreicher als in den entsprechenden, stark von periglazialen Komponenten durchsetzten Schottern des Donautales. Im Donautal könnte zur Hauptzeit der Lößanwehung außerdem noch eine andere Materialführung vorgelegen haben, als sie sich heute im Schotter darstellt; z. B. liegen an der Basis des Schotters große Weißjurablöcke, die dem Schotter selbst fehlen. Das Ende der Lößablagerung fällt mit dem beginnenden Eisrückzug zusammen; denn auf den zuerst trockengelegten, weil überhöhten würmeiszeitlichen Schotterflächen in Jungmoränennähe liegt noch eine dezimeterstarke Lößlage der ausklingenden Lößsedimentation.

Im niederbayerischen Donautal treten in einem schmalen Saum entlang dem Talrand flugsandreiche und sandstreifige Lösse als flußnahe Ablagerungen auf. Diese sandstreifigen Lösse fehlen dagegen in Jungmoränennähe (Ausnahme z. B. Schwaig - K. Brunnacker 1957a). Hier muß demnach wenigstens die Seitenerosion länger angehalten haben und dabei die randlichen Lößlager entfernt haben als im Donautal. Dieser Befund spricht außerdem für eine sehr schnelle Aufschotterung, denn andernfalls müßte die sandstreifige Lößfazies landeinwärts gewandert sein und dort im hangenden Teil des Lösses deutlicher hervortreten.

Wie bei Profil Kitzingen ausgeführt, besteht ein Zusammenhang zwischen Sedimentationsgeschwindigkeit und Kalkgehalt dahingehend, daß schnell abgelagerter Löß etwas kalkreicher ist als langsam abgelagerter. Auch der sandstreifige Löß entlang dem Donautal ist mit bis $4 \mathrm{~m}$ Mächtigkeit gegenüber dem normalen Löß mit $\mathrm{rd}$. 1,5-2 m wesentlich schneller aufgewachsen, weshalb sein Kalkgehalt (bei Pfelling) mit 40-45\% deutlich über dem des normalen Lösses mit dort rd. 30\% Kalk liegt. Da in der Flugsandkomponente bei Pfelling der Kalkgehalt nur 15-20\% beträgt, muß die Ursache des anormal hohen Kalkgehaltes in der sehr raschen Sedimentation des Lösses seine Ursache haben. Löß mit normaler Mächtigkeit, also mit etwas langsamerem Aufwuchs seiner Oberfläche, ist demnach bereits eines Teiles seines Kalkgehaltes während der Ablagerung beraubt worden. Der in Südbayern fast überall sehr geringmächtige Untere würmeiszeitliche Löß hat entsprechend diesem Befund einen Großteil seines Kalkgehaltes bereits als Folge seiner sehr langsamen Aufwuchsgeschwindigkeit verloren. Ein Teil des schon während der Ablagerung in Lösung gegangenen Kalkes wurde - abgesehen von dem an Ort und Stelle wieder ausgeschiedenen - im tieferen Profilbereich wieder ausgefällt (vgl. Hörlkofen - K. Brunnacker 1956b). In Kitzingen ist die Abhängigkeit der heutigen Kalkverteilung von der Sedimentationsgeschwindigkeit auch allein durch solche Umlagerungen innerhalb des Profiles erklärbar. Doch werden entsprechend den Beobachtungen in Südbayern auch hier die Verhältnisse so liegen, daß ein ursprünglich etwas kalkreicheres Material angeweht wurde, das je nach der Geschwindigkeit des Aufwuchses seiner Bodenoberfläche und entsprechend den im Laufe der Sedimentationszeit etwas wechselnden klimatischen Verhältnissen einen gewissen Kalkanteil verloren hat. Der gelöste Kalk wurde teilweise wieder ausgeschieden. Daher kommt die Aufkalkung im oberen Bereich des W I/II-Bodens. Das nämlich gilt für die Proben Nr. 15 und 16, die ablagerungsmäßig noch in die Zeit langsamer Sedimentation gehören, dem Kalkgehalt nach aber schon infolge dieser nachträglichen Aufkalkung zu dem darüber liegenden Abschnitt schnellerer Sedimentation überleiten. Bei diesen Vorgängen handelt es sich um eine an die Lößablagerung gekoppelte Bodenbildung. In Kitzingen war deren Tiefenwirkung - die Kalkverlagerungen innerhalb des Profiles zugrunde gelegt - deutlich geringer als in Hörlkofen.

Die von E. Schönhals (1952) gefundene Beziehung zwischen Kalkgehalt und Korngrößenzusammensetzung bei frischem Löß (hoher Kalkgehalt entspricht hohem Anteil 


\begin{tabular}{|c|c|c|c|c|c|c|c|c|c|c|c|}
\hline \multirow{3}{*}{ Nr. } & \multicolumn{10}{|c|}{ Korngrößenverteilung in $\mathrm{mm}(\%)$} & \multirow{3}{*}{$\begin{array}{c}\mathrm{CaCO}_{3} \\
(\%)\end{array}$} \\
\hline & \multicolumn{3}{|c|}{ Grobsand: } & \multicolumn{3}{|c|}{ Feinsand: } & \multicolumn{3}{|c|}{ Schluff: } & \multirow{2}{*}{$\frac{\mid \text { Rohton: }}{\langle 0.002}$} & \\
\hline & $2-1$ & $1-\overline{6}$ & $\begin{array}{l}0,6- \\
0,2\end{array}$ & $\begin{array}{l}0,2- \\
0,1\end{array}$ & $\begin{array}{c}0,1- \\
0,06\end{array}$ & $\begin{array}{l}0,06- \\
0,02\end{array}$ & $\begin{array}{l}0,02- \\
0,01\end{array}$ & $\begin{array}{l}0,01- \\
0,006\end{array}$ & $\begin{array}{l}0,006- \\
0,002\end{array}$ & & \\
\hline \multirow{2}{*}{2} & 0,1 & 0,2 & 0,6 & 0,8 & 3,2 & 37,3 & 21,2 & 4,6 & 7,6 & \multirow{2}{*}{24,4} & \multirow{2}{*}{14,9} \\
\hline & \multicolumn{3}{|c|}{0,9} & \multicolumn{3}{|c|}{41,3} & \multicolumn{3}{|c|}{33,4} & & \\
\hline \multirow{2}{*}{6} & 0,1 & 0,2 & 1,1 & 1,1 & 1,7 & 42,3 & 20,5 & 6,8 & 6,5 & \multirow{2}{*}{19,7} & \multirow{2}{*}{18,1} \\
\hline & \multicolumn{3}{|c|}{1,4} & \multicolumn{3}{|c|}{45,1} & \multicolumn{3}{|c|}{33,8} & & \\
\hline \multirow{2}{*}{10} & 0,3 & 0,5 & 1,2 & 1,0 & 2,2 & 43,5 & 20,2 & 7,6 & 5,7 & \multirow{2}{*}{17,8} & \multirow{2}{*}{21,0} \\
\hline & \multicolumn{3}{|c|}{2,0} & \multicolumn{3}{|c|}{46,7} & \multicolumn{3}{|c|}{33,5} & & \\
\hline \multirow{2}{*}{14} & 0,1 & 0,3 & 0,5 & 0,6 & 2,4 & 46,7 & 20,6 & 6,1 & 5,6 & \multirow{2}{*}{17,1} & \multirow{2}{*}{20,2} \\
\hline & \multicolumn{3}{|c|}{0,9} & \multicolumn{3}{|c|}{49,7} & \multicolumn{3}{|c|}{32,3} & & \\
\hline \multirow{2}{*}{19} & 0,2 & 0,4 & 1,0 & 0,7 & 3,2 & 41,8 & 21,2 & 7,1 & $6,0^{\circ}$ & \multirow{2}{*}{18,4} & \multirow{2}{*}{18,1} \\
\hline & & 1, & & & 45,7 & & & 34 &, 3 & & \\
\hline 24 & 0,1 & 0,5 & 0,8 & 0,7 & 2,6 & 43,2 & 22,5 & 7,0 & 6,4 & 16,3 & 20,1 \\
\hline 27 & & 1, & & & 46,5 & & & 35 &, 9 & 10,5 & \\
\hline 31 & 0,3 & 0,7 & 1,2 & 0,7 & 4,3 & 34,4 & 19,3 & 9,1 & 8,0 & 22,0 & 17,0 \\
\hline & & 2, & & & 39,4 & & & 36 &, 4 & & \\
\hline WI/II & 0,1 & 0,3 & 0,9 & 0,1 & 6,3 & 26,1 & 20,1 & 8,8 & 8,3 & 28,4 & 2,3 \\
\hline (A) & & 1, & & & 32,5 & & & 37 & ,2 & 28,4 & 2,3 \\
\hline
\end{tabular}

Tabelle II: Korngrößenverteilung und Kalkgehalte einiger Proben des Profils Kitzingen (vgl. Abb. 1)

Von Nr. 24 und 14 zu Nr. 10 steigt der Gehalt an feineren Korngrößen bei zunehmendem Kalkgehalt an. In Nr. 19, 6, 31, 2 und W I/II-(A) steigt der tonige Anteil in der angegebenen Reihenfolge an bei abnehmendem Kalkgehalt als Folge stärkerer Verwitterung; in Nr. 31 und 19 synsedimentär, in Nr. 6 und 2 infolge nacheiszeitlicher bzw. in W I/II-(A) infolge interstadialer Verwitterung. (Die Probe W I/II liegt in Profil $10-15 \mathrm{~cm}$ unterhalb der Nr. $31-$ K. Brunnacker 1956a). Die 1956a aus dem Oberen Löß untersuchte Probe ist ebenfalls bereits durch die Verwitterung etwas gestört.

an feineren Korngrößen) liegt in Kitzingen nur in einigen daraufhin untersuchten Proben vor (Tabelle II) - bei den von vornherein sehr geringen Unterschieden der Analysenwerte machen Fehlerquellen, die durch die Probenahme bzw. die Analysenmethode bedingt sind, sehr viel aus; dies muß berücksichtigt werden. Die Zusammenstellung der Tabelle II, aber auch der Tabelle I zeigt, daß außerdem der tonige Anteil des Lösses ansteigt, sobald etwas mehr Kalk weggeführt wurde (als Folge langsamerer Sedimentation oder nachträglicher Verwitterung). Vornehmlich wurde zuerst der in den feineren Korngrößen vorhandene Kalk gelöst und verlagert. Soweit er wieder mehr oder weniger weitgehend ausgeschieden wurde, dann vor allem in der Tonfraktion. Solange derartige Kalkausscheidungen im Endergebnis zum annähernd gleichen Kalkgehalt führten, wie er ursprünglich vorgelegen hat, stimmt das Verhältnis von Kalkgehalt zu Korngrößenverteilung noch (z. B. Tabelle I, $100-115 \mathrm{~cm}$ ). Wo aber etwas stärkere Kalkwegführung gegeben ist, die durch nachträgliche Aufkalkung nicht einigermaßen kompensiert werden konnte, stimmt das genannte Verhältnis nicht mehr (Tabelle I u. II). Bei verstärkter Wegführung von Kalk werden einmal innerhalb der Fraktion des Schluffes liegende kalkverkittete tonige Teilchen frei - auch die kalkverkitteten Rhizosolenien werden dabei z. T. zerstört, während die Schneckenschalen anfänglich bzw. bei schwa- 
chen Verlagerungen nicht oder nur wenig in Mitleidenschaft gezogen werden (in Hörlkofen sind die Schalen zerstört!). Außerdem wirkt sich, wie schon E. SchönhaLS (1952) betont, bei stärkerer Entkalkung eine Korngrößenverschiebung nach der feinkörnigeren Seite hin aus als Folge der Entfernung der die einzelnen Körnchen umhüllenden Kalkhäutchen. Erst wenn der Kalk vollständig weggeführt ist, setzt stärkere Silikatverwitterung und dabei auch Tonneubildung ein. Hierdurch kommt es zu weiterer Tonanreicherung im Bodenbereich. Einzelne Bodenhorizonte können durch Tonverlagerungen zusätzlich verändert werden (Parabraunerde).

Den Untersuchungen von E. SCHöNhals (1952) lagen Einzelproben zugrunde. Dementsprechend zeigen die von ihm vorgeführten Zusammenstellungen eine gewisse Streubreite, die durch örtliche Unterschiede oder durch Differenzierungen innerhalb der einzelnen Profile, wie in Kitzingen gezeigt, bedingt sind. Ein Vergleich der für den Oberen Löß in Kitzingen ermittelten Werte mit denen von Estenfeld bestätigt den Befund von E. Schönhals, wobei die Estenfelder Proben als etwas kalk- und tonreicher sich an die von Kitzingen annähernd anschließen.

Die Kalkverteilung im Löß bietet damit Hinweise auf die periglazialen Bodenbildungsvorgänge; so zeigt das Profil Hörlkofen wesentlich tiefer reichende Kalkumlagerungen und Zerstörung der Schneckenschalen im Gegensatz zu Kitzingen. Abgesehen von den Profilen im feuchtesten Bereich der Lößablagerung in Südbayern, wozu Hörlkofen gehört, ist die Kalkverlagerung im Löß normalerweise während seiner Ablagerung und während seiner Sedimentationspausen (W I/II) in Südbayern wegen des kontinentaleren Klimas weniger stark wirksam und wird wegen der an sich höheren Kalkgehalte auch nicht so deutlich wie in Mainfranken.

\section{Schlußfolgerungen}

Wahrscheinlich wurde gegen Ende der Lößbildung die Sedimentation etwas verlangsamt, wie sie auch zu Beginn noch nicht gleich mit voller Stärke eingesetzt hat. Die Unterschiede in der heutigen Bodenausbildung zwischen Löß und älterem Schwemmlöß im Donautal (K. BrunNaCKer 1957b) lassen sich jedoch dadurch allein nicht erklären. Es kommt die verstärkte Entkalkung zu Beginn des Spätglazials noch hinzu, der sich wieder eine Verlangsamung der Entkalkung bis zum Beginn der Wiederbewaldung anschloß, und darauf folgte auf diesem so verbreiteten Material die warmklimatische Bodenbildung (als Parabraunerde).

Die Bedeutung der Vegetation für das Festlegen des Lößstaubes lag weniger in deren Eigenschaft als Staubfänger begründet, weshalb der Löß an ausgesprochen nassen vegetationsgünstigen Standorten auch nicht mächtiger ist als in den umgebenden trockeneren und damit vegetationsärmeren Bereichen. Die Vegetation hat vor allem durch Wurzelatmung und bei ihrem Abbau Kohlensäure geliefert, die die Lösung des Kalkes begünstigte. Soweit dieser Kalk dann wieder ausgeschieden wurde, hat er leichte, an Wurzelgängen auch stärkere, Verfestigungen des Lösses bewirkt und damit den Löß fixiert; denn der gelöste Kalk wurde nicht nur verlagert und im tieferen Untergrund durch Austrocknung, zu der auch die winterliche Gefrornis gehört, sondern auch im Oberflächenbereich durch sommerlichen Wasserentzug wieder ausgeschieden. Wo die Vegetation nicht aufkommen konnte, also auf Sand, Ton oder in Bereichen stärkerer Solifluktion, ebenso in für die Vegetation zu kalten oder zu trockenen Gegenden, wurde deshalb der Staub immer wieder erneut aufgenommen. Die Transgression der von den nach O exponierten Hängen ausgehenden Lößablagerung bedarf jedoch noch weiterer Erklärungen.

Hinsichtlich der Lößmächtigkeiten ist jeweils zu prüfen, ob es sich um talrandnahe Bildungen handelt (hoher Kalkgehalt!) oder um die die einzelnen Landschaften kennzeichnenden Durchschnittsmächtigkeiten (mit entsprechendem Durchschnittskalkgehalten). Bei geringeren Mächtigkeiten kann eine ganz langsame und deshalb kalkarme 
Ablagerung vorliegen, es kann sich aber auch um eine aus der Zeit der ausklingenden Lößablagerung stammende Bildung handeln. Die Ausdeutung dünner Lößdecken, die noch dazu durch die nacheiszeitliche Verwitterung völlig verändert und häufig auch durch junge Erosion beeinflußt sind, bereitet deshalb einige Schwierigkeiten. Beispielsweise zeigen die zahlreichen, aber verbreitungsmäßig sehr kleinen Lößlager Mittelfrankens (vgl. H. Loeber 1952), daß in Mittelfranken praktisch überall Lößstaub hingeweht wurde. Fast überall wurde er aber durch den Wind wieder aufgenommen und damit bzw. durch Solifluktion weiter vertragen. Lediglich an einzelnen Stellen konnte er liegen bleiben, weil dort von Anfang an die entsprechenden Voraussetzungen vorgelegen haben. Die Lößmächtigkeit ist in diesen einzelnen mittelfränkischen Lagern etwas geringer als in Mainfranken. Der geringe Kalkgehalt des Lösses in Mittelfranken wird. deshalb sowohl durch kalkarme Komponenten im Ausgangsmaterial und durch anscheinend etwas gröbere Körnung wie auch durch eine verhältnismäßig langsame Aufwuchsgeschwindigkeit des Lösses in diesem Gebiet bedingt sein.

Insgesamt zeigt sich aber, daß die Zeiten der Lößbildung zumindest nicht überall einen vollariden Charakter besessen haben.

\section{$\mathrm{Schrift} \mathrm{tum}$}

Brunnacker, K.: Das Lößprofil in Kitzingen (Unterfranken). Ein Beitrag zur Chronologie des Paläolithikums. - Germania 34, S. 3-11, 1956 (1956a). - - Das Lößprofil von Hörlkofen/Obb. - Neues Jb. Geol. Paläontol. Mh. 1956, S. 424-433, Stuttgart 1956 (1956b). - - Die Geschichte der Böden im jüngeren Pleistozän in Bayern. - Geol. Bavarica, München 1957 (1957a). - - Geologische Karte von Bayern 1 : 25000 Nr. 7142 Straßkirchen mit Erläuterungen. - München 1957 (1957b).

Brunnacker, M. \& Brunnacker, K.: Die Molluskenfauna einiger Lößprofile im Donautal. Geol. Bl. NO-Bayern 6, S. 91-100, Erlangen 1956.

Guenther, E. W.: Feinstratigraphische Studien III. Methoden zur Untersuchung von Lössen. Neues Jb. Geol. Paläontol., Mh. 1953, S. 97-111, Stuttg. 1953 (1953a). - - Feinstratigraphische Untersuchung von Lößprofilen. - Actes du IV Congrés International du Quaternaire 1953, S. 1-7, Rom 1953 (1953b). - - Feinstratigraphische Untersuchung eines Lößprofils von Murr (Landkreis Ludwigsburg). - Eiszeitalter und Gegenwart 4/5, S. 147-157, Öhringen 1954.

Löber, H.: Zur Kenntnis der Lößvorkommen in Mittelfranken. - Diss. Univ. Erlangen 1932. SchöNHALs, E.: Gesetzmäßige Beziehungen zwischen Körnung und Kalkgehalt des Lösses und die Erkennung von Verwitterungszonen mit Hilfe der typischen Streubereiche. Geol. Jb. 66, S. 291-304, Hannover 1952. - - Gesetzmäßigkeiten im Feinaufbau von Talrandlössen mit Bemerkungen über die Entstehung des Lösses. Eiszeitalter und $\mathrm{Ge}$ genwart 3, S. 191-236, Óhringen 1953. - - Kennzahlen für den Feinheitsgrad des Lösses. - Ebendort 6, S. 133-147, 1955.

Manuskr. eingeg. 4. 1. 1957.

Anschrift des Verf.: Dr. K. Brunnacker, Bayr. Geolog. Landesamt, München 22, Prinzregentenstraße 26. 